
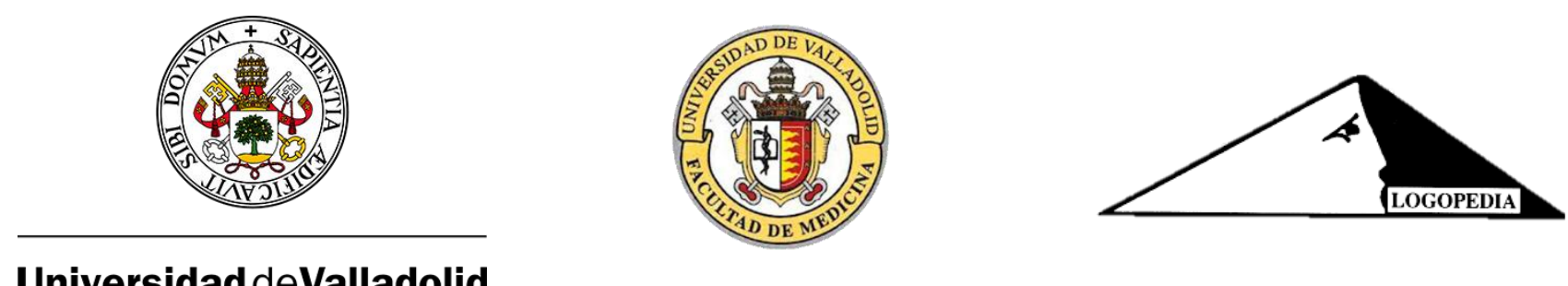

Universidad deValladolid

TRABAJO DE FIN DE GRADO

Grado en Logopedia

\title{
SITUACIÓN DE LA LOGOPEDIA EN LA PROVINCIA DE SORIA: VISIÓN PROFESIONAL Y SOCIAL
}

\author{
Autora: MARÍA LAFUENTE LAFUENTE \\ Tutora: ROSA BELÉN SANTIAGO PARDO
}

CURSO 2019/2020 


\section{AGRADECIMIENTOS}

Llegar a este punto en el que se culmina la realización de este Trabajo de Fin de Grado no ha sido fácil, y este no habría sido el resultado sin la ayuda de todas las personas que han colaborado y me han animado a conseguirlo a lo largo de este camino.

En primer lugar, quería agradecer a todas las personas, pacientes, familiares y logopedas que han colaborado con sus opiniones y experiencias en este trabajo, por su inversión de tiempo y disponibilidad para hacerlo, gracias a lo cual se le ha podido otorgar veracidad y autenticidad, y por invitarme en su día a reflexionar sobre la realidad de la logopedia en la provincia de Soria, provincia que necesita que la escuchen, y que con este trabajo he podido darla un poco más de voz.

También destacar de forma especial a mi tutora, Rosa Belén Santiago Pardo, por apostar por este trabajo desde el primer momento, por su paciencia infinita, sus buenos consejos y su constancia durante todo este proceso, intentando sacar lo mejor en cada momento, tanto a nivel personal como profesional, a lo largo de estos cuatro años.

Finalmente, dar las gracias a mi familia y amigos, que han sido un pilar fundamental en esta etapa que termina, por animarme a seguir y apoyarme en todo momento. 


\section{RESUMEN}

El presente trabajo desarrolla un análisis en profundidad de la situación real de la logopedia en la provincia de Soria desde diferentes puntos de vista, con el objetivo de exponer el progreso y la realidad que vive la profesión en pequeños territorios, caracterizados por la falta de recursos sociales e institucionales. Para comprender cómo se ha llegado al punto actual, es necesario conocer previamente el momento demográfico que vive la provincia, y que juega un papel muy importante en el desarrollo de servicios tales como el de la logopedia, que, como profesión joven, aún lucha por hacerse un hueco en los diferentes ámbitos que abarcan su campo de actuación. Como resultado de este estudio, se han agrupado los datos sobre la presencia del logopeda en los servicios de la provincia de Soria en los que tiene cabida este profesional, así como en comparación con otras provincias de Castilla y León, como son Segovia y Valladolid. Asimismo, también se ha otorgado carácter social a dichos resultados con la realización de 19 cuestionarios directos a logopedas, familiares y pacientes que forman parte del servicio de logopedia en la provincia de Soria, cuyas opiniones se han utilizado para la contrastación de los datos cuantitativos, permitiendo así la elaboración de conclusiones que reflejan la realidad de la situación actual.

Palabras clave: logopedia, provincia de Soria, situación, logopedas, población.

\section{ABSTRACT}

The present work develops an in-depth analysis of the real situation of speech therapy in the province of Soria (Spain) from different points of view. This is done in order to present the progress and the actuality that the profession is experimenting in small areas that are defined by the lack of social and institutional resources. To understand how they got to this point, it is necessary to know beforehand the demographic situation of the province since it plays a very important role in the performance of social services such as speech therapy. As a young profession, speech therapy still struggles to make a name of itself in the areas covered by its field of action. As a result of this study, data on the presence of speech therapists in the services of the province of Soria where this professional can be found has been gathered. A comparison with other provinces of Castilla y León such as Segovia and Valladolid has been made as well. Additionally, a social dimension has been given to those results throughout 19 surveys directed to speech therapists, family members and patients that are part of the speech therapy service in the province of Soria. Their opinions have been 
used to contrast the quantitative data, thus allowing the elaboration of conclusions that reflect the reality of the current situation.

Key words: speech therapy, province of Soria, situation, speech therapist, population. 


\section{ÍNDICE}

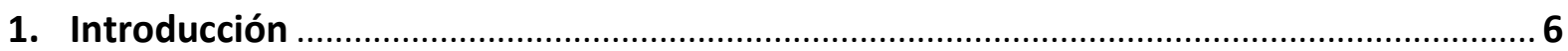

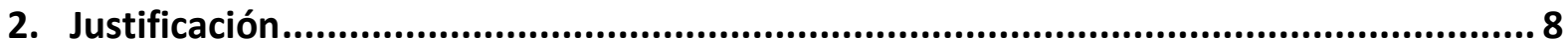

2.1. La provincia de Soria y su situación demográfica .......................................................8

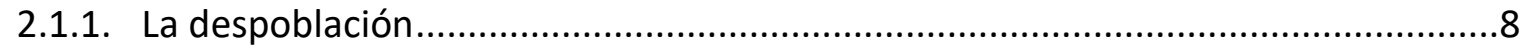

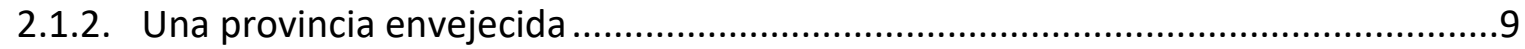

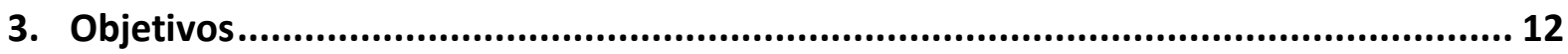

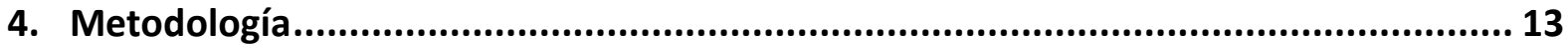

4.1. Metodología para el estudio social a través de entrevistas .....................................13

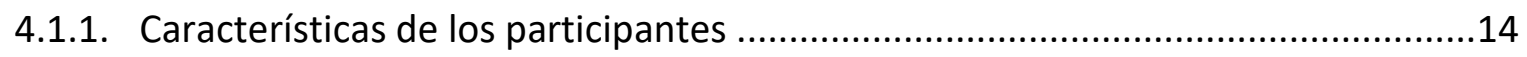

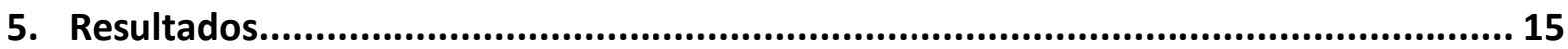

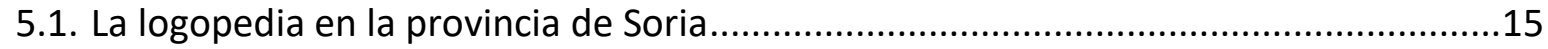

5.1.1. La actualidad de la logopedia en la provincia de Soria .......................................15

5.1.2. Comparativa con otras provincias: Segovia y Valladolid .....................................17

5.2. La situación de la logopedia en la provincia de Soria desde la perspectiva social .......23

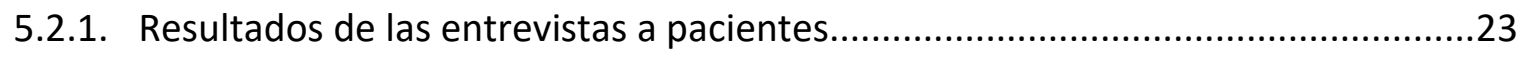

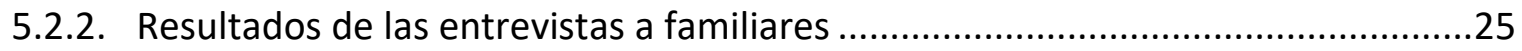

5.2.3. Resultados de las entrevistas a logopedas ........................................................26

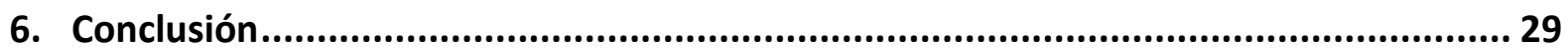

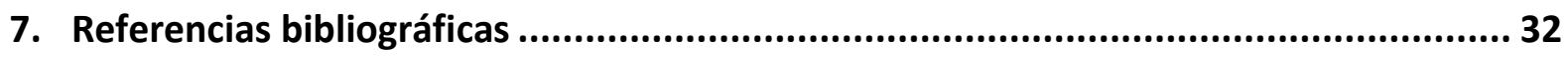

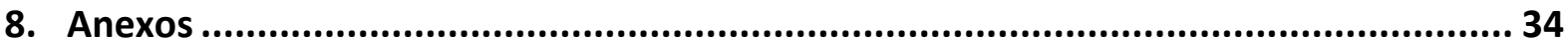

Anexo 1: Evolución de la población de la provincia de Soria (1900-2019)........................34

Anexo 2: Número de habitantes mayores de 65 años en la provincia de Soria a 1 de

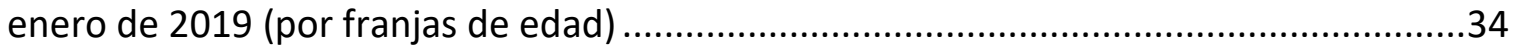

Anexo 3: Localización de los centros de logopedia indicados en el Registro General de Centros, Servicios y Establecimientos Sanitarios de las provincias de Soria, Segovia y

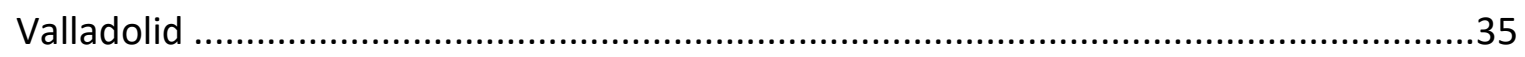

Anexo 4: Modelos de entrevista para el estudio social ....................................................36

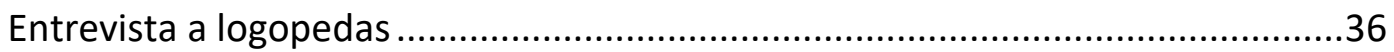

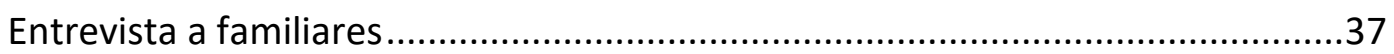

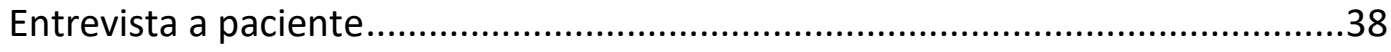




\section{INTRODUCCIÓN}

Desde la implantación en España de los estudios oficiales de Diplomatura de Logopedia en la Universidad de Valladolid y la Universidad Complutense de Madrid en el curso 1992-93, nuestra profesión ha ido in crescendo en todos sus niveles y se está consiguiendo que ocupe el lugar que le corresponde dentro de la Sanidad española, gracias a la gran labor que los docentes, investigadores y profesionales han llevado a cabo en estos últimos años para dar visibilidad a la logopedia como profesión sanitaria, necesaria en la rehabilitación del gran número de patologías que abarca nuestro campo de actuación.

Sin embargo, cuando se habla del desarrollo de la logopedia en España, en la mayoría de las ocasiones, se hace referencia al crecimiento de la profesión a nivel nacional y en comparación con otros países, sobre todo de Europa y América, a los que tomamos como ejemplo en este largo camino hacia el reconocimiento de la figura del logopeda en nuestra sociedad. Por ello, lo que se pretende con este trabajo es que por un momento se deje de englobar este progreso como un todo para centrar la mirada y reflexionar sobre la situación en la que se encuentra la logopedia en los pequeños territorios, como la provincia de Soria, que, caracterizados por un abandono institucional, carecen de los recursos suficientes para que este impulso se produzca al mismo nivel que en otras regiones.

La elección de este tema para realizar este Trabajo de Fin de Grado surge tras la realización de las prácticas curriculares de último curso en uno de los centros de logopedia que encontramos en la ciudad de Soria, en el que se tuvo la oportunidad de conocer un poco más de cerca cómo se desarrolla el servicio allí. A raíz de esta experiencia, y del auge expositivo acerca de la situación de la España Vaciada, nos pareció muy interesante y necesario mostrar también esta realidad de nuestra profesión, y qué mejor forma de hacerlo que de la mano de los protagonistas que participan en ella como son los logopedas que trabajan en la provincia de Soria, los pacientes y sus familiares.

Con todo ello, este trabajo se va a dividir en dos partes. En la primera parte, de carácter cuantitativo, se muestran los datos reales de la situación y regulación de la logopedia en la provincia de Soria, y en comparación con otras provincias de Castilla y León, en este caso, con las provincias de Segovia y Valladolid. La elección de centrar la información en estas regiones se debe a que Segovia es la provincia que sigue a Soria en número de 
habitantes, y Valladolid, además de ser la capital de la comunidad, es la ciudad donde se oferta el Grado en Logopedia, a través de la Universidad de Valladolid. La segunda parte se construye a partir de una investigación cualitativa, a partir de la cual se busca conocer la visión de la situación de la logopedia en la provincia de Soria que tienen los profesionales, los pacientes y los familiares de pacientes que forman y/o han formado parte de este servicio en la provincia de Soria.

No obstante, es preciso señalar que, no se han encontrado trabajos previos acerca de este tema tan específico, por lo que no podemos contrastar nuestro trabajo con el de otros autores. 


\section{JUSTIFICACIÓN}

A lo largo de este trabajo, se va a exponer la información recogida en relación al desarrollo actual de la logopedia en la provincia de Soria, pero antes es necesario establecer unas bases y conocer el punto en el que se encuentra a día de hoy esta región. Por ello, nos centraremos previamente en conocer la provincia de Soria y su situación demográfica, con el desarrollo del tema de la despoblación y el envejecimiento, para, posteriormente, enfocar nuestra mirada en el estado actual de la logopedia en Soria, y también en comparación otras provincias de Castilla y León, como son Segovia y Valladolid.

\subsection{LA PROVINCIA DE SORIA Y SU SITUACIÓN DEMOGRÁFICA}

\subsubsection{LA DESPOBLACIÓN}

Soria es una provincia perteneciente a la comunidad de Castilla y León y situada en la parte norte del interior de España. Actualmente, viven en ella 88.636 habitantes (Instituto Nacional de Estadística, 2019) y cuenta con una densidad de población de 8,6 habitantes $/ \mathrm{km}^{2}$, estando en octava posición del ranking de las zonas más despobladas de Europa (Oficina Europea de Estadística, 2020), pero ¿por qué se utilizan tanto hoy en día los términos "despoblación" o "España Vaciada" para referirse a territorios como el de la provincia de Soria? Para comprender la gravedad de la situación demográfica que se vive en esta región, es necesario hacer una pequeña referencia a la evolución que ha tenido la provincia durante el siglo XX, y, más concretamente, en la segunda mitad de siglo.

Alrededor del año 1910, la provincia contaba con una población de 156.354 habitantes, repartida en 345 municipios, de los que 15 tenían una población superior a 1.000 habitantes y sólo uno presentaba menos de 100 habitantes inscritos en su padrón (Antequera, 2018). Esto se mantuvo estable durante las primeras décadas del siglo XX, hasta llegar a alcanzar la cifra de 161.182 habitantes totales en la provincia en el año 1950 (INE, s.f.). A partir de ese momento, se inició un brusco descenso poblacional, que ha llegado hasta nuestros días, causado por la migración de los habitantes de la provincia a los núcleos urbanos que la rodean, como Madrid, Bilbao o Zaragoza, en los que en esos años se produjo un importante desarrollo industrial que no llegó a realizarse en Soria, donde la agricultura tampoco permitía la introducción de nueva mano de obra (Gerencia de Asistencia Sanitaria de Soria, s.f.) (Anexo 1). 
Este descenso progresivo se estabilizó con la llegada del siglo XXI y, actualmente, la provincia está formada por 183 municipios, de los cuales 117 tienen menos de 100 habitantes, y 67 de ellos menos de 50. Asimismo, solo 11 municipios superan los 1000 habitantes, uno de ellos la capital, en la que habitan 39.398 personas, lo que corresponde a un $44,4 \%$ de la población total de la provincia (INE, 2019). Pero, sobre todo, hay un dato comparativo que refleja muy bien el fenómeno de la despoblación en Soria, y es que cuando en 1910 el 75\% de la población se concentraba en los primeros 88 municipios con más habitantes (Antequera, 2018); más de un siglo después, en el año 2019, solo con los 10 primeros municipios con más población se consigue alcanzar dicho porcentaje (INE, 2019).

A pesar de esto, el pronóstico, no tan a largo plazo, sigue la misma línea de lo vivido hasta ahora, ya que debido a la falta de políticas realistas que hagan hincapié en la potenciación de la industria, entre otras cosas, los jóvenes se ven obligados a emigrar a otras ciudades en las que pueden tener mayores perspectivas de futuro, hecho que está siendo reivindicado por plataformas ciudadanas como Soria iYa! (http://soriaya.org/) o la asociación Jóvenes de Castilla y León en Madrid (https://www.jovenescyl.org/). Además, la centralización de la población en los municipios más importantes de la provincia como la capital, ha supuesto que en ella también se concentren tanto administraciones estatales y autonómicas como la atención hospitalaria y otros servicios, entre los que se encuentra la logopedia, lo cual se tratará posteriormente (Antequera, 2018).

\subsubsection{UNA PROVINCIA ENVEJECIDA}

Este fenómeno migratorio, tanto a la capital como a otras regiones, junto con la escasez de oportunidades ha generado que, sobre todo en los pequeños núcleos de población, predomine actualmente un alto índice de envejecimiento que condena a muchos municipios a la desertización a medio y corto plazo (Pinilla y Sáez, 2017), debido a la falta de un relevo generacional, sufrida por 69 de los 183 municipios de la provincia, los cuales no tienen habitantes con una edad inferior a los 15 años (INE, 2019). 
Según datos del INE (2019), 22.747 personas de la provincia de Soria tienen más de 65 años, lo que corresponde a un 25,6\% del total de la población (Anexo 2). Además, el Índice de Envejecimiento ${ }^{1}$ de esta región se encuentra en un 194,15\%, frente al 122,88\% del total nacional; y existe una Tasa de Dependencia de la población mayor de 64 años $^{2}$ del 41,60\%, mientras que este dato en España se sitúa en un 29,93\%.

En cuanto a la pirámide de población de la provincia a 1 de enero de 2019, observamos que hay una clara diferencia entre las tres franjas de edad representadas: jóvenes (0-29 años), adultos (30-64 años) y ancianos (más de 65 años), como se puede comprobar en la Figura 1.

\section{Figura 1}

Pirámide de Población de la Provincia de Soria del año 2019

Provincia de Soria 2019

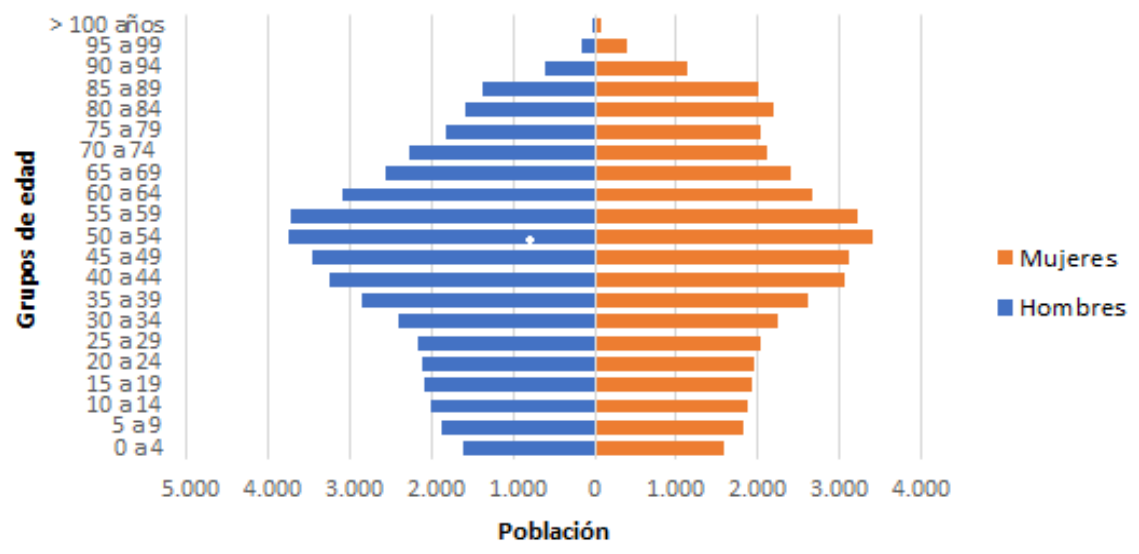

Fuente: elaboración propia a partir de datos del INE a 1 de enero de 2019.

Existe un predominio de la población adulta, sobre todo de la comprendida en el rango de 40 a 65 años, y, a partir de esto, hay una llamativa diferencia en los cambios de franja, tanto a la de los ancianos como a la de los jóvenes. A medida que se va subiendo en los rangos de edades, hay una disminución progresiva de las personas con más edad, pero se mantienen con un número bastante elevado de habitantes con respecto a los adultos. Sin embargo, al hablar de los jóvenes, se observa que el descenso en la población es bastante brusco, sobre todo a partir de los 29 años, produciéndose, al igual que con los adultos

\footnotetext{
${ }^{1}$ Porcentaje que representa la población mayor de 64 años sobre la población menor de 16 años, a 1 de enero del año 2019.

2 Tasa que relaciona la población inactiva o económicamente dependiente frente a la población potencialmente activa. Se obtiene a través del cociente entre los individuos mayores de 64 años dividido entre la población potencialmente activa.
} 
mayores, una reducción progresiva paralela al descenso en los rangos que representan a los habitantes de menor edad. Esto nos deja dos conclusiones principales: uno de los problemas más importantes que presenta el territorio es la falta de un relevo generacional que condena a la provincia a la desaparición de numerosos núcleos rurales, y que la provincia de Soria es el claro ejemplo de una población envejecida. 


\section{OBJETIVOS}

OBJETIVO GENERAL: Analizar la situación real de la logopedia en la provincia de Soria. OBJETIVOS ESPECÍFICOS:

- Explicar la situación actual que vive la provincia de Soria en relación a la despoblación y el envejecimiento.

- Detallar la regulación profesional de la logopedia en la provincia de Soria y compararla con otras provincias de Castilla y León, en concreto, con las provincias de Valladolid y Segovia.

- Conocer la visión de los profesionales sobre el momento actual del servicio de logopedia en la provincia de Soria.

- Conocer la visión de los familiares y los pacientes sobre la logopedia y las repercusiones que se producen en su vida diaria el tener que acudir al logopeda en la provincia de Soria. 


\section{METODOLOGÍA}

Durante la elaboración de este trabajo se ha llevado a cabo un importante proceso de búsqueda de información a través de diferentes fuentes, lo cual ha resultado un proceso laborioso debido a la dificultad de encontrar datos concretos sobre esta temática, ya que no existen trabajos anteriores sobre este tema, y por la falta de datos accesibles de forma pública.

Se han utilizado diferentes bases de datos, como Google Académico/Avanzado o Dialnet, a partir de las cuales se han agrupado diferentes artículos e informes demográficos, así como se han consultado los datos recogidos en el Instituto Nacional de Estadística (INE) y en la Oficina Europea de Estadística (EUROSTAT) sobre población y envejecimiento.

Para la agrupación de la información relacionada con la logopedia, se han consultado una amplia lista de páginas web de asociaciones y de organismos oficiales como el SACYL, la Junta de Castilla y León, el Colegio Profesional de Logopedas de Castilla y León o el Ministerio de Sanidad, Consumo y Bienestar social. También se ha contactado a través de correo electrónico con diferentes entidades de las nombradas anteriormente con el fin de recabar la información que no se encuentra de forma pública. Asimismo, cabe mencionar que también ha sido necesario consultar portales no oficiales para el acceso a la información sobre las residencias de personas mayores, ya que en la actualidad no existe un registro oficial en el que se agrupen los centros pertenecientes a este ámbito en Castilla y León.

\subsection{METODOLOGÍA PARA EL ESTUDIO SOCIAL A TRAVÉS DE ENTREVISTAS}

Con el fin de conocer la visión social de la logopedia en la provincia de Soria, se han elaborado tres modelos de entrevistas (Anexo 4) destinadas a logopedas, pacientes y familiares de pacientes que han acudido o están acudiendo a logopedia en dicha provincia. Es necesario mencionar que debido a la prohibición de desplazamiento por la pandemia COVID-19 no se han podido realizar de forma presencial, por lo que la forma de contacto con las personas colaboradoras ha sido a través de la red social Whatsapp o por correo electrónico, lo cual ha dificultado la obtención de mayor cantidad de respuestas por falta de colaboración o por la imposibilidad de contactar con algunas personas. 
En total han sido contestadas, para su posterior análisis, 19 entrevistas, de las cuales ocho han sido realizadas por pacientes, tres por familiares y ocho por logopedas.

\subsubsection{CARACTERÍSTICAS DE LOS PARTICIPANTES}

- Grupo 1: pacientes. Se han realizado ocho entrevistas a pacientes que han recibido tratamiento logopédico, todos ellos en la provincia de Soria, por diversas patologías. Las edades de las personas que forman este grupo de entrevistados oscilan desde los 21 a los 53 años. Durante el tratamiento, seis de los participantes vivían en municipios ajenos a la capital, en concreto, cinco de ellos en El Burgo de Osma (a 57 km de Soria) y uno en Navaleno (a $47 \mathrm{~km}$ de Soria). Las dos personas restantes residían en la ciudad de Soria durante el tratamiento.

- Grupo 2: familiares. Se han realizado tres entrevistas a personas del entorno de pacientes que han acudido o acuden a logopedia. En este caso, el 100\% de las personas que han cumplimentado este modelo de entrevista viven en un municipio distinto a la capital, concretamente en El Burgo de Osma, y corresponden a familiares directos (padre/madre) de dichos pacientes. Cabe mencionar que ninguno de los tres cuestionarios se ha realizado a familiares de personas incluidas en el grupo 1.

- Grupo 3: logopedas. En total han sido ocho las entrevistas realizadas a diferentes logopedas, de los cuales siete trabajan actualmente como logopedas en Soria, y uno de ellos figura como colegiado en la provincia ya que procede de la misma, pero actualmente trabaja en Madrid. Los profesionales que se encuentran en Soria ejercen la logopedia en diferentes ámbitos:

- Cinco logopedas trabajan en un centro privado-concertado al que acuden pacientes derivados de la seguridad social.

- Un logopeda trabaja en un centro que pertenece al sector privado.

- Un logopeda trabaja en una asociación.

Tras agrupar las respuestas a los cuestionarios se ha procedido a su análisis del cual se han obtenido una serie de resultados y conclusiones desarrollados en los siguientes apartados de este trabajo. 


\section{RESULTADOS}

\subsection{LA LOGOPEDIA EN LA PROVINCIA DE SORIA}

A pesar de las circunstancias que rodean a la provincia, uno de los servicios que se ha ido abriendo camino durante las últimas décadas es el de la logopedia y, aunque de forma más progresiva y tardía que en otros lugares de España, está inmersa en una evolución creciente, la cual esperemos que se siga produciendo en los próximos años.

Uno de los factores más importantes por los que es necesario que se siga originando este desarrollo es el del envejecimiento, ya que, debido al alto índice que existe y que va a ir en aumento a medida que pasen los años, se convierte en una de las principales poblaciones diana en las que va a ser fundamental el trabajo del logopeda para que, junto con la labor de otros profesionales, consigan para estas personas el máximo nivel de autonomía posible en sus actividades cotidianas, y, en consecuencia, en su funcionamiento global (González, Mendizábal, Jimeno, y Sánchez, 2019). En relación a esto, es esencial que el logopeda realice labores tanto preventivas en personas mayores sanas, estimuladoras y rehabilitadoras en personas mayores con deterioro cognitivo leve, o labores para frenar la evolución negativa de las personas con demencia, para tratar de reducir en la menor medida posible los efectos que los cambios en los ámbitos biológico, psicológico y social generan en este tipo de población.

\subsubsection{LA ACTUALIDAD DE LA LOGOPEDIA EN LA PROVINCIA DE SORIA}

A raíz de los datos que se exponen en la página web de Colegio Profesional de Logopedas de Castilla y León (http://logopedascyl.es/) se conoce que hay actualmente 11 logopedas colegiados en la provincia de Soria. Esto se traduce en que en esta región hay un logopeda por cada 8.057 habitantes, situándose de manera significante por encima de la media de los datos del total de la comunidad, que se corresponden con un logopeda por cada 5.105 habitantes. A partir de estas cifras, y desde el punto de vista de las personas mayores de 65 años como población susceptible a intervención logopédica, se obtiene que en Soria existe 1 logopeda por cada 2.067 habitantes con más de 65 años, también superando la media de Castilla y León, la cual se sitúa en 1 logopeda por cada 1.242 habitantes. 
Para conocer los datos sobre las clínicas de logopedia en la provincia de Soria, acudimos al Registro General de Centros, Servicios y Establecimientos Sanitarios (http://regcess.mscbs.es/), regulado por el Ministerio de Sanidad, Consumo y Bienestar social, en el cual observamos que hay inscritos tres centros de logopedia en la provincia. En relación a esto, se ha llevado a cabo una búsqueda propia en la que se ha encontrado que existen otros dos centros que presentan logopedia, por lo que en conjunto suman cinco centros de logopedia en total en la provincia, de los cuales cuatro son privados y uno es privado-concertado, que cuenta con un convenio con el SACYL, al que acuden, además de pacientes relacionados con el ámbito privado como en el resto de centros, aquellos que son derivados de la Seguridad Social.

Por otra parte, no se cuenta con la presencia fija de logopeda en ninguno de los dos hospitales que forman en Complejo Hospitalario de Soria (Hospital Santa Bárbara y Hospital Virgen del Mirón), pero, ante la llegada de los pacientes que presentan patologías logopédicas, son las logopedas del centro concertado las que acuden a realizar la primera valoración e intervención necesaria en ese ámbito.

En cuanto a las personas mayores, se han recogido datos sobre los centros residenciales a partir del portal de información Inforesidencias.com (www.inforesidencias.com), debido a la falta de un registro oficial, y se ha comprobado que en ninguna de las 37 residencias de la provincia de Soria se ofrece el servicio de logopedia, ni, en su defecto, algún tipo de recurso que esté orientado a potenciar la comunicación y el lenguaje a través de terapias de estimulación y/o rehabilitación en este tipo de población.

En lo que respecta a las asociaciones, en Soria existe la sede de siete asociaciones (SACYL, 2019) relacionadas con síndromes o trastornos en los que la figura del logopeda es importante para su bienestar global. Estas asociaciones son las siguientes:

- Asociación de Familiares de Enfermos de Alzheimer Soria.

- Asociación Parkinson Soria.

- FADISO (Federación de Asociaciones con Discapacidad Física, Orgánica y Sensorial de Soria).

- ASAMIS (Asociación soriana de Ayuda a las Personas con Discapacidad Intelectual y sus Familias) 
- ASPACE Soria (Asociación de Parálisis Cerebral).

- Asociación Autismo Soria.

- ASOEM (Asociación Soriana de Esclerosis Múltiple)

De estas, tres de ellas cuentan con servicio de logopedia (Asociación de Familiares de Enfermos de Alzheimer, Asociación Parkinson y ASPACE), de las cuales en solo una (ASPACE) hay un logopeda fijo todo el año. En el resto, son los profesionales de los centros privados las que acuden a dar sesiones algunas horas a la semana.

En el ámbito de los Servicios Sociales, como se indica en la página web de la Junta de Castilla y León (https://www.jcyl.es/), en la capital se sitúa la Unidad de Valoración y Atención a Personas con Discapacidad, dependiente de las Gerencias Territoriales de Servicios Sociales. En ella es donde se presta el Servicio de Atención Temprana, en el cual la figura del logopeda es fundamental. En Soria actualmente hay un logopeda trabajando en este servicio, aunque se desconoce si de forma fija o itinerante, como en el caso de las asociaciones.

\subsubsection{COMPARATIVA CON OTRAS PROVINCIAS: SEGOVIA Y VALLADOLID.}

El siguiente punto va a tratar los datos de logopedia correspondientes a otras provincias de Castilla y León con el fin de comparar la situación en ellas con la de la provincia de Soria, desarrollada anteriormente. Se han escogido las provincias de Segovia y Valladolid por las siguientes razones:

- Segovia por ser la provincia que está por encima de Soria en número de habitantes (Soria: 88.636 habitantes/Segovia: 153.129 habitantes).

- Valladolid, además de por ser la capital de la comunidad y la que más habitantes tiene (519.546 habitantes), es el centro de la logopedia en Castilla y León al encontrarse en la ciudad el Grado en Logopedia de la Facultad de Medicina de la UVa. 
En primer lugar, en el Directorio de Colegiados del Colegio Profesional de Logopedas de Castilla y León (http://logopedascyl.es/), se indican los siguientes datos sobre los logopedas colegiados en las tres provincias, representados en la Tabla 1:

\section{Tabla 1}

Logopedas Colegiados en las Provincias de Soria, Segovia y Valladolid

\begin{tabular}{cc}
\hline PROVINCIAS & LOGOPEDAS COLEGIADOS \\
\hline Soria & 11 logopedas \\
Segovia & 27 logopedas \\
Valladolid & 164 logopedas \\
\hline
\end{tabular}

Fuente: elaboración propia a partir de datos del Colegio Profesional de Logopedas de Castilla y León.

Se aprecia una diferencia clara entre ellas, que se justifica al existir un paralelismo con el aumento en el número de habitantes de cada provincia. Pero al calcular el dato de logopedas por habitante, se obtiene lo siguiente: encontramos un logopeda por cada 5.671 habitantes en la provincia de Segovia, y un logopeda por cada 3.167 habitantes en la provincia de Valladolid, situando a Segovia ligeramente por encima y a Valladolid con bastante diferencia por debajo de lo que respecta a la media de Castilla y León (un logopeda por cada 5.105 habitantes). Además, en el dato de logopedas por habitantes mayores de 65 años, en Segovia hay un logopeda por cada 1.285 adultos mayores y, en lo que corresponde a Valladolid, un logopeda por cada 726, también ambas por debajo de la media de Castilla y León (un logopeda por cada 1.242 habitantes). Esto refleja que en Soria (un logopeda por 8.057 habitantes/un logopeda por 2.067 habitantes mayores de 65 años) podría existir una falta de logopedas con respecto a estas provincias concretas.

En lo que respecta a las clínicas de logopedia, los datos que ofrece el Registro General de Centros, Servicios y Establecimientos sanitarios ${ }^{3}$ (http://regcess.mscbs.es/) son los que se exponen a continuación en la Tabla 2.

\footnotetext{
${ }^{3}$ En este caso se han tomado los datos oficiales, centrados exclusivamente en lo que se indica en el Registro General de centros, servicios y establecimientos sanitarios que proporciona el Ministerio de Sanidad, Consumo y Bienestar social.
} 


\section{Tabla 2}

Número de Centros de Logopedia en las Provincias de Soria, Segovia y Valladolid

\begin{tabular}{cc}
\hline PROVINCIAS & NÚMERO DE CENTROS \\
\hline Soria & 3 \\
Segovia & 2 \\
Valladolid & 21 \\
\hline
\end{tabular}

Fuente: elaboración propia a partir de datos del Registro General de centros, servicios y establecimientos sanitarios.

Llama la atención que en la provincia de Segovia únicamente existen dos centros de logopedia registrados, lo que supondría una gran desventaja con respecto a otras provincias debido a su elevado número de habitantes, por lo que habría que comprobar si este dato refleja fielmente la realidad o, por el contrario, existen centros que no se encuentran registrados. Sin embargo, otro aspecto que cabe destacar es que tanto en Segovia como en Valladolid existen centros en municipios distintos a la capital, siendo Soria la única provincia en la que no hay centros de logopedia en ningún municipio, sino que solo encontramos logopedas en la misma ciudad de Soria.

En el ámbito hospitalario regulado por el SACYL, y en cuya página web se indica, de las tres provincias solo hay presencia de logopedas en la capital, Valladolid, donde cuentan con logopedas presenciales en los dos hospitales públicos de la ciudad (Hospital Universitario Río Hortega y Hospital Clínico Universitario). En el sector privado, Segovia cuenta con logopeda en el Hospital Recoletas Segovia, perteneciente al Grupo Recoletas. Por lo que, en este aspecto, Soria también se queda atrás sin ningún logopeda presencial en los dos hospitales que forman su complejo hospitalario (Hospital Santa Bárbara y Hospital Virgen del Mirón).

Por otro lado, encontramos a partir de la información que nos proporcionan la página web de los portales de información que registran estos centros, que en las residencias de personas mayores de las tres provincias hay una escasez muy llamativa de la presencia del logopeda entre sus servicios. Ni en la provincia de Soria, que cuenta con 37 residencias, ni en la provincia de Segovia, que tiene un total de 43 residencias, ofrecen un servicio de logopedia para sus residentes. Valladolid es la provincia puntera en este caso, puesto que sí 
encontramos logopeda en cinco de las 101 residencias de esta región. A pesar de esto, destaca la poca inclusión de la logopedia dentro de estos centros en las tres provincias analizadas, puesto que se deja de lado uno de los factores más importantes que contribuyen al bienestar global de estas personas, como es la comunicación, así como otro tipo de patologías asociadas también susceptibles a la intervención del logopeda.

Desde el punto de vista de las asociaciones (SACYL, 2019), Valladolid es la que destaca con más sedes de asociaciones para personas que pueden presentar alguna patología logopédica, lo cual está directamente relacionado con su posición como capital de comunidad, así como por el número de habitantes que presenta. La relación de esta con las provincias de Soria y Segovia en lo que se refiere a las asociaciones está indicada en la tabla 3:

Tabla 3

Presencia de Servicio de Logopedia en las Asociaciones de las Provincias de Soria, Segovia y Valladolid

\begin{tabular}{|c|c|c|c|c|c|c|}
\hline & \multicolumn{2}{|c|}{ SORIA } & \multicolumn{2}{|c|}{ VALLADOLID } & \multicolumn{2}{|c|}{ SEGOVIA } \\
\hline & Asociaciones & $\begin{array}{c}\text { Servicio de } \\
\text { logopedia }\end{array}$ & Asociaciones & $\begin{array}{l}\text { Servicio de } \\
\text { logopedia }\end{array}$ & Asociaciones & $\begin{array}{l}\text { Servicio de } \\
\text { logopedia }\end{array}$ \\
\hline ALZHEIMER & $\begin{array}{l}\text { Asociación de } \\
\text { Familiares y } \\
\text { enfermos de } \\
\text { Alzheimer } \\
\text { Soria }\end{array}$ & No & $\begin{array}{l}\text { Asociación } \\
\text { de Familiares } \\
\text { y enfermos } \\
\text { de Alzheimer } \\
\text { Valladolid }\end{array}$ & No & $\begin{array}{l}\text { Asociación } \\
\text { de Familiares } \\
\text { y enfermos } \\
\text { de Alzheimer } \\
\text { Segovia }\end{array}$ & No \\
\hline ATAXIA & $\begin{array}{l}\text { No hay } \\
\text { asociación }\end{array}$ & - & FEDAES & $\begin{array}{l}\text { Sí (de forma } \\
\text { online) }\end{array}$ & $\begin{array}{l}\text { No hay } \\
\text { asociación }\end{array}$ & - \\
\hline AUTISMO & $\begin{array}{l}\text { Asociación } \\
\text { Autismo Soria }\end{array}$ & Sí & $\begin{array}{l}\text { Asociación } \\
\text { Autismo } \\
\text { Valladolid }\end{array}$ & Sí & $\begin{array}{l}\text { Asociación } \\
\text { Autismo } \\
\text { Segovia }\end{array}$ & Sí \\
\hline DAÑO CEREBRAL & $\begin{array}{l}\text { No hay } \\
\text { asociación }\end{array}$ & - & $\begin{array}{l}\text {-ICTIA } \\
\text { (ASPAYM) } \\
\text {-Camino }\end{array}$ & $\begin{array}{l}\text {-Sí } \\
\text { •No }\end{array}$ & $\begin{array}{l}\text { No hay } \\
\text { asociación }\end{array}$ & - \\
\hline $\begin{array}{l}\text { DISCAPACIDAD } \\
\text { AUDITIVA }\end{array}$ & $\begin{array}{l}\text { No hay } \\
\text { asociación }\end{array}$ & - & $\begin{array}{l}\text {-APERSORVA } \\
\text {-APSAVA } \\
\text {-ASPAS }\end{array}$ & $\begin{array}{l}\text {-Desconocido } \\
\text {-Desconocido } \\
\text {-Sí }\end{array}$ & ASEFAS & Desconocido \\
\hline
\end{tabular}




\begin{tabular}{|c|c|c|c|c|c|c|}
\hline $\begin{array}{l}\text { DISCAPACIDAD } \\
\text { FÍSICA }\end{array}$ & \begin{tabular}{|l} 
No hay \\
asociación
\end{tabular} & - & ASPAYM & No & $\begin{array}{l}\text { Asociación } \\
\text { Coordinada } \\
\text { de Personas } \\
\text { con } \\
\text { Discapacidad } \\
\text { Física de } \\
\text { Castilla y } \\
\text { León }\end{array}$ & No \\
\hline $\begin{array}{l}\text { DISCAPACIDAD } \\
\text { INTELECTUAL }\end{array}$ & $\begin{array}{l}\text {-ASAMIS } \\
\text {-FADISO }\end{array}$ & -No & $\begin{array}{l}\text { - } \text { Fundación } \\
\text { Personas } \\
\text { - ASPRONA } \\
\text { - ASCEDIS } \\
\text { - Centro } \\
\text { Especial } \\
\text { Padre Zegrí } \\
\text { - Centro San } \\
\text { Juan de } \\
\text { Dios } \\
\text { - Red } \\
\text { Círculos }\end{array}$ & $\begin{array}{l}\text { •Sí } \\
\text { •Sí } \\
\text { •No } \\
\text { •Sí } \\
\text { •Sí } \\
\text { •No }\end{array}$ & APADEFIM & No \\
\hline $\begin{array}{l}\text { ESCLEROSIS } \\
\text { MÚLTIPLE }\end{array}$ & ASOEM & No & $\begin{array}{l}\text { No hay } \\
\text { asociación }\end{array}$ & - & ASGEM & Sí \\
\hline LARINGECTOMÍAS & $\begin{array}{l}\text { No hay } \\
\text { asociación }\end{array}$ & - & ASOVALL & Sí & $\begin{array}{l}\text { Asociación } \\
\text { Provincial de } \\
\text { Laringectomi } \\
\text { zados }\end{array}$ & Sí \\
\hline $\begin{array}{l}\text { PARÁLISIS } \\
\text { CEREBRAL }\end{array}$ & ASPACE & Sí & ASPACE & Sí & ASPACE & Sí \\
\hline PARKINSON & $\begin{array}{l}\text { Asociación } \\
\text { Parkinson } \\
\text { Soria }\end{array}$ & Sí & APARVAL & Sí & $\begin{array}{l}\text { Asociación } \\
\text { Parkinson } \\
\text { Segovia }\end{array}$ & Sí \\
\hline $\begin{array}{l}\text { SÍNDROME DE } \\
\text { DOWN }\end{array}$ & $\begin{array}{l}\text { No hay } \\
\text { asociación }\end{array}$ & - & $\begin{array}{l}\text { DOWN } \\
\text { Valladolid }\end{array}$ & Sí & ASIDOS & Sí \\
\hline TDAH & $\begin{array}{l}\text { No hay } \\
\text { asociación }\end{array}$ & - & AVATDAH & No & $\begin{array}{l}\text { No hay } \\
\text { asociación }\end{array}$ & - \\
\hline
\end{tabular}

Fuente: elaboración propia.

Al observar la tabla 3 comprobamos que en Valladolid encontramos que de las 20 asociaciones de la provincia en las que tiene cabida la figura del logopeda, en 12 sí que ofrecen este servicio. En el caso de Segovia, existe servicio de logopedia en seis de las 11 asociaciones que se sitúan en esta provincia. Por lo que se concluye que, en esta comparativa, Soria es la provincia en la que las personas con patología susceptibles de requerir intervención logopédica menos posibilidades tienen de conseguir este servicio a través de asociaciones. 
Por último, en lo que respecta al Servicio de Atención Temprana que se encuentra en la Unidades de Valoración y Atención a Personas con Discapacidad, se desconocen los datos del número de logopedas concretos que trabajan en este ámbito en las provincias de Segovia y de Valladolid, pero, a partir de información remitida por el Servicio de Autonomía Personal y atención a Personas con Discapacidad, regulado por los Servicios Sociales, se determina que el número de profesionales de logopedia varía en función a la población que se atiende. Como se ha indicado anteriormente, en Soria existe un logopeda, pero en otras provincias con mayor volumen de intervención están trabajando actualmente hasta cinco o seis logopedas. Por otra parte, se conoce que en las tres provincias a las que nos referimos en este punto del trabajo existe al menos una Unidad de Valoración y Atención a Personas con Discapacidad, en las que trabajan logopedas.

Además, para que los servicios proporcionados por este ámbito se extiendan también al medio rural, desde la Gerencia Territorial de Servicios Sociales existen acuerdos con los municipios de más de 20.000 habitantes y con ayuntamientos y Entidades Colaboradoras dependientes del tercer sector. Estos datos se indican en la tabla 4.

\section{Tabla 4}

Relación entre las Unidades de Valoración y Atención con las Entidades Colaboradoras y Ayuntamientos en las Provincias de Soria, Segovia y Valladolid

\begin{tabular}{ccc}
\hline PROVINCIAS & $\begin{array}{c}\text { UNIDADES DE } \\
\text { VALORACIÓN Y ATENCIÓN }\end{array}$ & $\begin{array}{c}\text { AYUNTAMIENTOS Y } \\
\text { ENTIDADES } \\
\text { COLABORADORAS }\end{array}$ \\
\hline Soria & 1 & - \\
Segovia & 1 & 1 \\
Valladolid & 3 & 2 \\
\hline
\end{tabular}

Fuente: tabla proporcionada por el Servicio de Autonomía Personal y atención a Personas con Discapacidad, dependiente de las Gerencias Territoriales de los Servicios Sociales.

Como se refleja en la tabla 4, Valladolid es la provincia con más Unidades de Valoración y Atención a Personas con Discapacidad (3) y con más Entidades Colaboradoras (2), que apoyan el trabajo de dichas unidades en otros puntos de la provincia. En el caso de Segovia, encontramos una Unidad y una Entidad Colaboradora dentro de la provincia. Por lo que Soria es la única provincia que el trabajo de las Unidades de Valoración y todos los 
servicios que se llevan a cabo en estas se encuentra únicamente en la capital, sin ninguna Entidad que lleve esta asistencia a otras zonas del medio rural que rodean a la ciudad de Soria, evidenciando, una vez más, la centralización existente tanto del servicio de logopedia como de las ayudas sociales que proporcionan este tipo de organizaciones.

\subsection{LA SITUACIÓN DE LA LOGOPEDIA EN LA PROVINCIA DE SORIA DESDE LA PERSPECTIVA SOCIAL}

Todos estos datos objetivos desarrollados en los apartados anteriores nos ayudan a hacernos una idea concreta de cuál es la situación actual de la logopedia en la provincia de Soria desde diferentes perspectivas. Sin embargo, para poder otorgar a estos datos un carácter más realista, es fundamental conocer qué opinan las personas que forman parte de la profesión día a día, es decir, los logopedas que trabajan en la provincia de Soria, sus pacientes y los familiares de estos pacientes, los cuales tienen un papel fundamental dentro de la intervención logopédica en muchos de los casos.

Para ello, se lleva a cabo un estudio social a partir de diferentes entrevistas, cuyos resultados se van a exponer a continuación, con el que se busca complementar la información recogida, verificando la realidad que nos aporta los datos, así como conocer otros aspectos que profundizan en el análisis como las necesidades existentes o la repercusión que se produce en la vida de los pacientes y familiares que acuden al logopeda en la provincia de Soria.

Cabe mencionar que, en relación a las entrevistas a los logopedas, los resultados que se van a desarrollar representan únicamente a una parte de los profesionales de logopedia que trabajan en la provincia, debido a que no ha sido posible establecer contacto con todos.

\subsubsection{RESULTADOS DE LAS ENTREVISTAS A PACIENTES}

El primer dato que llama a atención sobre los resultados de las entrevistas realizadas a ocho pacientes que han acudido a logopedia en la provincia de Soria, es que cinco de los ocho entrevistados no conocían el servicio anteriormente y, los tres que sí que lo conocen, se debe a que personas de su entorno habían acudido. Esto indica que, aunque esta sea una muestra pequeña de personas, hay un desconocimiento general por parte de la población de 
la existencia de la figura del logopeda, ya que, si no es porque alguien cercano ha acudido a logopedia, no hay conocimiento sobre nuestra profesión. En relación a esto, al narrar su experiencia yendo al logopeda, muchos de los pacientes relatan que al principio "no veían el motivo por el que acudir" pero que, a medida que iba transcurriendo el tratamiento se daban cuenta de la importancia del trabajo que realizan los profesionales y de lo necesario que es para muchas patologías. De esta forma, encontramos que no solo no se conoce al logopeda, sino que tampoco se tiene constancia de las funciones o campos de actuación en los que tiene cabida este profesional.

Por otra parte, en cuanto al servicio de logopedia y su organización, la mayoría de los pacientes comentan que, a nivel general y desde su experiencia, existe una buena regulación, destacando que apenas existe lista de espera a la hora de empezar un tratamiento, lo cual supone un gran beneficio de cara al posible pronóstico de una patología. Sin embargo, señalan algunas necesidades y/o aspectos de mejora enfocados al desarrollo de la logopedia en la provincia de Soria. En primer lugar, todos los pacientes a los que se les ha realizado la entrevista indican que la localización de los centros supone en muchas ocasiones un hándicap a la hora de llevar a cabo la rehabilitación logopédica, sobre todo para aquellas personas que viven en municipios ajenos a la capital, ya que el tener que realizar varios desplazamientos a lo largo de la semana, de una hora y media cada día aproximadamente en el caso de los entrevistados, para poder acudir a las sesiones genera muchas dificultades a la hora de compaginarlo con su vida laboral y familiar. Además, la escasa comunicación a través del transporte público de la capital con el resto de la provincia supone también una limitación de horarios que no siempre es fácil de coordinar con los propios centros. Otro aspecto muy comentado por los participantes es la presencia de la figura del logopeda en la Seguridad Social, ya que consideran que sería muy necesario que existieran consultas de logopedia dentro de los Centros de Salud de los diferentes puntos de la provincia, tanto para que aumentara el número de profesionales y de localidades en las que se pudiera acceder a este servicio, como para proporcionar más facilidades en lo que respecta al plano económico, puesto que creen que no todas las personas que precisen del servicio de un logopeda puedan permitirse pagar tanto el tratamiento, debido a la continuidad que requiere, como los propios viajes necesarios para poder acudir. 
En definitiva, aunque exista un buen funcionamiento interno del servicio, la mayoría de los pacientes opina que, a día de hoy, la logopedia es un servicio precario en la provincia de Soria debido a la falta de profesionales, la ausencia de centros en diferentes puntos de la provincia $y$, de forma general, porque es "menos conocida, menos accesible y menos reconocida".

\subsubsection{RESULTADOS DE LAS ENTREVISTAS A FAMILIARES}

Siguiendo la línea de los resultados en las entrevistas a los pacientes, en lo que respecta a las tres entrevistas realizadas a familiares encontramos que dos de ellos sí que conocían el servicio de logopedia y uno no. Al igual que con el grupo anterior, los familiares que afirman conocerlo indican que es debido a que algunas personas cercanas a ellos recibieron tratamiento logopédico anteriormente, y no por conocimiento de la profesión.

Por otro lado, y puesto que todas las personas entrevistadas del grupo de familiares corresponden a padres y madres cuyos hijos acuden a logopedia actualmente, señalan que, de la misma forma que en el caso de los pacientes, la mayor dificultad a la que tienen que hacer frente es la del desplazamiento. En las respuestas comentan que asistir a las sesiones de logopedia supone, además de la involucración de toda la familia, la dedicación del 100\% del tiempo de varias tardes a la semana, debido a que tienen que viajar a Soria, lo que se traduce, entre otras cosas, en la disminución de las horas de estudio de sus hijos o dificultades en cuanto a la conciliación laboral y/o familiar. Este es un factor que se repite en las tres entrevistas ya que, según indican, "hay personas que puntualmente no pueden acompañar a sus familiares el $\mathbf{1 0 0 \%}$ de las veces", lo que puede suponer que, en determinadas ocasiones, el paciente falte a la sesión. Asimismo, destacan la buena comunicación que existe entre el servicio y los familiares, lo que facilita el mantenimiento de la motivación hacia el tratamiento.

Por estas razones, los tres familiares entrevistados creen que la logopedia es un servicio precario en la provincia de Soria, justificándolo con la falta de centros de logopedia en más puntos de la provincia, así como con la necesidad de implantación de los logopedas dentro de la Seguridad Social para así cumplir con el objetivo de facilitar el acceso a este servicio, así como de otorgarle la visibilidad de la cual carece fuera de la capital a día de hoy. 


\subsubsection{RESULTADOS DE LAS ENTREVISTAS A LOGOPEDAS}

En primer lugar, encontramos algunos datos que indican la presencia de una gran disparidad entre los diferentes centros: mientras que, actualmente, al centro privado acuden unos 10 pacientes al año y 21 a la asociación, en el centro privado-concertado se atiende a una media de 350 pacientes al año, cifra que está directamente relacionada con el convenio que presenta este centro con la Seguridad Social. Por otra parte, el número de pacientes que acuden a cada centro también está asociado al número de logopedas que trabajan en ellos, ya que tanto en el centro privado como en la asociación se cuenta con la presencia de un logopeda, mientras que el centro privado-concertado está integrado por cinco logopedas.

Otro aspecto que llama la atención es el número de años que cada logopeda lleva trabajando en la provincia, puesto que en las respuestas nos indican que cuatro de ellas han estado ejerciendo como logopedas en Soria durante 26, 25, 18 y 17 años; y tres durante 7 meses, 3 años y 3 años y medio. Encontramos una diferencia de 14 años que lleva a señalar la presencia de dos generaciones de logopedas, y con la que podemos deducir lo siguiente:

- Durante estos años ha habido poca población soriana que se haya interesado en formarse como logopedas, lo cual podría deberse a una falta de visibilidad de la profesión y estaría íntimamente relacionado con el escaso número de logopedas que aparecen colegiados en la provincia de Soria actualmente.

- No ha habido profesionales que hayan llegado a la provincia con objetivos de permanecer en ella a largo plazo o no han tenido oportunidad de cumplirlos por la falta de empleo como logopedas, más allá de instalar una clínica propia.

Sin embargo, se observa que en estos últimos años ha habido un aumento en el número de profesionales de la provincia, lo que podría estar relacionado con un incremento de la demanda por parte de otros colectivos como asociaciones.

Por otra parte, todos los profesionales a los que se les ha hecho la entrevista han indicado que, además de trabajar en su centro/asociación, acuden a otros ámbitos a ejercer como logopedas como a los centros hospitalarios, otras asociaciones de pacientes o centros escolares, ya sea actualmente o en algún momento de su trayectoria profesional. Por lo que, aunque no exista un gran número de logopedas en la provincia de Soria, las necesidades que 
puedan surgir en otros campos de actuación donde se requiere la presencia de estos profesionales estarían cubiertas gracias a la labor que realizan los que a día de hoy desarrollan su trabajo en esta región.

En lo que respecta a la forma de organización del servicio de logopedia en Soria los logopedas mencionan ciertas ventajas y algunos aspectos deficitarios. Entre las ventajas, varios destacan que, al ser una provincia pequeña, hay mayor proximidad entre los profesionales lo que favorece la coordinación entre el personal sanitario y el servicio de rehabilitación. Además, aunque no exista Unidad de Logopedia en los propios hospitales, la atención en el complejo hospitalario se inicia prácticamente en el momento de la demanda, además, estos ofrecen a los profesionales externos que acuden a realizar las valoraciones logopédicas de los pacientes todo el material necesario para llevarlas a cabo facilitando así las labores de realización. Sin embargo, también refieren que ambas ventajas son un arma de doble filo, ya que, en muchas ocasiones, debido a la falta de concienciación de las administraciones con respecto a las labores del logopeda, se producen menos derivaciones de las que son necesarias y, cuando estas se producen, el $100 \%$ del material que se utiliza en las sesiones con estos pacientes tiene que ser aportado por el centro. Otro de los aspectos que señalan los profesionales, y que ya se ha comentado a lo largo de este trabajo, es la falta de centros especializados/organizaciones especializadas en patologías específicas (daño neurológico adquirido, ELA, Síndrome de Down...), y que, en el caso de que los hubiera, contaran con logopedas en sus plantillas.

Todos estos aspectos, de una forma u otra, pueden llegar a influir en el tratamiento de los pacientes, lo que se suma a algunas dificultades que las personas que acuden a logopedia en la provincia de Soria tienen que hacer frente. Entre las diferentes repercusiones de los puntos deficitarios que caracterizan al servicio, los profesionales indican que, en el caso de los pacientes que acuden a logopedia por derivación del SACYL, una vez que acaban las sesiones concertadas, el paciente se queda sin apoyos, y, si presenta una patología crónica que necesita continuidad en el tratamiento logopédico, en muchos casos no puede acudir tampoco a asociaciones donde sí se le ofrecería la ayuda requerida, también por la falta de estas. Por otro lado, la falta de concienciación general del sistema sanitario a la hora de cuándo y en qué casos realizar las derivaciones al logopeda hace que se deje de lado un punto muy importante de cara a la intervención logopédica: la 
prevención; lo cual genera que colectivos como el de las personas mayores, que corresponde a una gran parte de la población en la provincia de Soria, no tengan acceso a este tipo de tareas de estimulación lingüística que puede ser anticipatoria de problemas mayores a largo plazo. Por otro lado, existen varias dificultades que se repiten entre los pacientes que acuden a realizar su tratamiento logopédico a Soria, y que, en ocasiones, dificultan la continuidad en la rehabilitación. La principal, según señalan los logopedas, es la de la distancia y el desplazamiento. El hecho de que los únicos centros de logopedia se sitúen en la capital hace que muchas personas que no viven en ella tengan problemas a la hora de desplazarse, tanto por falta de medios (poco servicio de transporte público o no posesión de vehículo propio) como por la meteorología, dependiendo de la época del año; o por dificultades en la conciliación familiar o laboral.

No obstante, aunque existan todavía muchos avances que realizar, la mayoría de los profesionales opinan que, a nivel general, el servicio de logopedia en la provincia de Soria funciona bien ya que se dispone de varios centros, asociaciones específicas y asistencia en los centros escolares, lo que supone que, a día de hoy, la demanda esté cubierta. 


\section{CONCLUSIÓN}

Tras analizar en profundidad y desde diferentes perspectivas la situación de la logopedia en la provincia de Soria, se ponen en manifiesto las siguientes conclusiones:

- Las personas mayores son una de las principales vías de actuación del logopeda dentro de la provincia de Soria debido a la gran densidad de población mayor de 65 años que presenta. Sin embargo, a día de hoy, no existe ningún tipo de plan, tanto por parte del Sistema General de Salud como por las instituciones especializadas, como las residencias, que contemple la implantación de la logopedia como un servicio necesario de cara al tratamiento de las posibles patologías que aparecen en el envejecimiento activo, y no solo en el plano comunicativo, cuya estimulación y/o rehabilitación es fundamental para fomentar la autonomía de este tipo de población, si no por otras dificultades que puedan presentar como problemas deglutorios, entre otros. Asimismo, no solo existe esta necesidad cuando ya hay establecido un tratamiento activo para la rehabilitación de una patología, sino también en el plano preventivo, el cual todavía se tiene menos en cuenta a pesar de su importancia sobre todo de cara al futuro próximo en el que nos vamos a encontrar con una sociedad envejecida. Además, esto se extrapola también a otras provincias de la comunidad de Castilla y León, en las que, al igual que en Soria, el alto índice de envejecimiento es una realidad, pero en ellas tampoco se ha producido la inclusión del logopeda como pieza fundamental para el bienestar de esta población.

- El desarrollo de la logopedia en la provincia de Soria se ha visto frenado por varios factores, entre los que se encuentra la centralización general de los principales servicios que se ofrecen a la ciudadanía como consecuencia de la migración de la población desde las zonas rurales a la capital de provincia. Esto ha generado que todo el progreso que ha tenido la profesión en estos últimos años se haya producido únicamente en la ciudad de Soria, lo que conlleva que hayan aparecido una serie de dificultades para las personas del resto de la región a la hora de someterse a un tratamiento logopédico, entre las que se encuentran la necesidad de desplazamiento y el problema de la conciliación laboral y/o familiar. Otro factor que también ha afectado considerablemente dentro del avance de la logopedia, y que se ha demostrado en la comparativa con las provincias de Segovia y Valladolid, es la norma 
que establece que la cantidad de servicios de una provincia va en función de la cantidad de habitantes que viven en ella. Esto provoca que en los servicios que proporciona el Estado en los que tiene cabida la figura del logopeda, la presencia de este sea mínima $\mathrm{o}$, en ocasiones, inexistente, tal y como ocurre en ámbito hospitalario o en el Servicio de Atención Temprana incluido en las Unidades de Valoración y Atención. Este aspecto también se ve reflejado en organizaciones privadas sin ánimo de lucro como las asociaciones destinadas a pacientes y familiares las cuales, además de tener dificultades para instaurarse en Soria debido a la poca población existente con patologías determinadas, carecen de recursos suficientes para ofrecer algunos servicios a sus integrantes, entre los que se encuentra la logopedia en muchas de ellas y esto supone, para los colectivos que lo necesitan, una falta en este tipo de apoyos que sí que es posible encontrar en otras provincias con mayor población y recursos.

- La principal necesidad que actualmente presenta la logopedia de la provincia de Soria para que se sigan produciendo avances es la visibilidad de la profesión, lo cual se ha ido poniendo en evidencia tanto a lo largo del desarrollo de este trabajo como a partir de la experiencia propia como estudiante de logopedia y habitante de esta provincia. En primer lugar, la centralización del servicio en la capital, de lo cual se ha hablado anteriormente, genera que exista, por parte de la población que habita en las zonas rurales, un desconocimiento general de la profesión y de las funciones que el logopeda desempeña, con ideas que se basan en los estereotipos que nos rodean y que están muy asociados a la figura del Maestro de Audición y Lenguaje el cual sí que está más extendido en los colegios de la provincia. Todo ello, favorecido por la juventud de la profesión, ha tenido como consecuencia la ausencia de logopedas de la provincia de Soria a lo largo de muchos años, lo cual se ve reflejado actualmente en el número de logopedas colegiados en Soria, que es el más inferior en comparación con el resto de las provincias de Castilla y León. Por lo que, si se impulsara, con la ayuda de los diferentes organismos oficiales, la necesidad del trabajo de los logopedas, sobre todo en una población como la que habita en la provincia de Soria, aumentaría el interés por la formación en este campo y, de forma paralela, los profesionales en la provincia, favoreciendo así el aumento de los 
recursos laborales en lugares como las asociaciones o las zonas rurales, y la inclusión de los mismos en otros ámbitos como en el de las residencias de personas mayores.

Finalmente señalamos que, a pesar de las dificultades con las que tiene que lidiar la logopedia en la provincia de Soria, debido a la falta de recursos que se otorgan como consecuencia de la situación de despoblación que sufre el territorio, en los últimos años se ha producido un aumento de los profesionales y personas interesadas en cubrir estas necesidades en el sector, que, junto con la labor que llevan realizando los logopedas desde que iniciaron su labor en esta provincia hace ya 25 años, se ha promovido que el desarrollo de la logopedia en Soria no se haya frenado en ningún momento, asegurando, de esta forma, un futuro prometedor para la profesión en la provincia, para el que se ha intentado contribuir con la realización de este trabajo, que da voz a los datos y opiniones que se habían mantenido en la sombra hasta el día de hoy, y para el que se va a colaborar en el futuro próximo con la finalización de los estudios del Grado en Logopedia. 


\section{REFERENCIAS}

Antequera, E. (2018, Febrero). Un análisis breve de las características de la despoblación en la provincia de Soria. Cuadernos de Ordenación del Territorio, 57-61.

EUROSTAT. (30 de abril de 2020). How closely do people live together in your region? https://ec.europa.eu/eurostat/en/web/products-eurostat-news/-/DDN-20200430-1

Gerencia de Asistencia Sanitaria de Soria. (s. f.). La Sanidad de Soria. http://www.sanidadsoria.es/Organizacion/O Demografica

González Martín. E. et alii (2019). Manifestaciones lingüísticas en personas mayores: el papel de la intervención logopédica en el envejecimiento sano y patológico. Revista de Investigación en Logopedia, 9(1), 1-19. http://dx.doi.org/10.5209/RLOG.60770

Instituto Nacional de Estadística. (2019). Índice de Envejecimiento por provincia. https://www.ine.es/iaxiT3/Tabla.htm?t=1489\&L=0

Instituto Nacional de Estadística. (2019). Población por provincias y sexo. https://www.ine.es/jaxiT3/Tabla.htm?t=2852\&L=0

Instituto Nacional de Estadística. (2019). Población por provincias, edad (grupos quinquenales, Españoles/Extranjeros, Sexo y Año. https://www.ine.es/jaxi/Tabla.htm?path=/t20/e245/p08/\&file=03002.px\&L=0 Instituto Nacional de Estadística. (2019). Población por sexo, municipios y edad (grupos quinquenales). https://www.ine.es/jaxi/Tabla.htm?path=/t20/e245/p05/a2019/10/\&file=00042001.p $\underline{x \& L=0}$ 
Instituto Nacional de Estadística. (2019). Soria: población por municipios y sexo.

https://www.ine.es/jaxiT3/Tabla.htm?t=2896\&L=0

Instituto Nacional de Estadística. (2019). Tasa de Dependencia de la población mayor de 64 años, por provincia. https://www.ine.es/jaxiT3/Tabla.htm?t=1492\&L=0

Instituto Nacional de Estadística. (s. f.). Población (1950) por provincias, edad y sexo. https://www.ine.es/jaxi/Tabla.htm?path=/t20/e245/p06/I0/\&file=1950.px\&L=0

Pinilla, V., y Sáez. L. A. (2017). Del pasado reciente al futuro próximo. Enseñanzas de la evolución demográfica y de las políticas planteadas sobre la despoblación en Cuenca, Soria y Teruel. Red de Áreas Escasamente Pobladas del Sur de Europa (SSPA).

Portal de residencias de la tercera edad. www.inforesidencias.com

SACYL. Asociaciones de Pacientes y Familiares. https://www.saludcastillayleon.es/AulaPacientes/es/asoc-pacientes SACYL. Hospitales de Castilla y León. https://www.saludcastillayleon.es/es/hospitales 


\section{ANEXOS}

ANEXO 1: EVOLUCIÓN DE LA POBLACIÓN DE LA PROVINCIA DE SORIA (1900-2019)

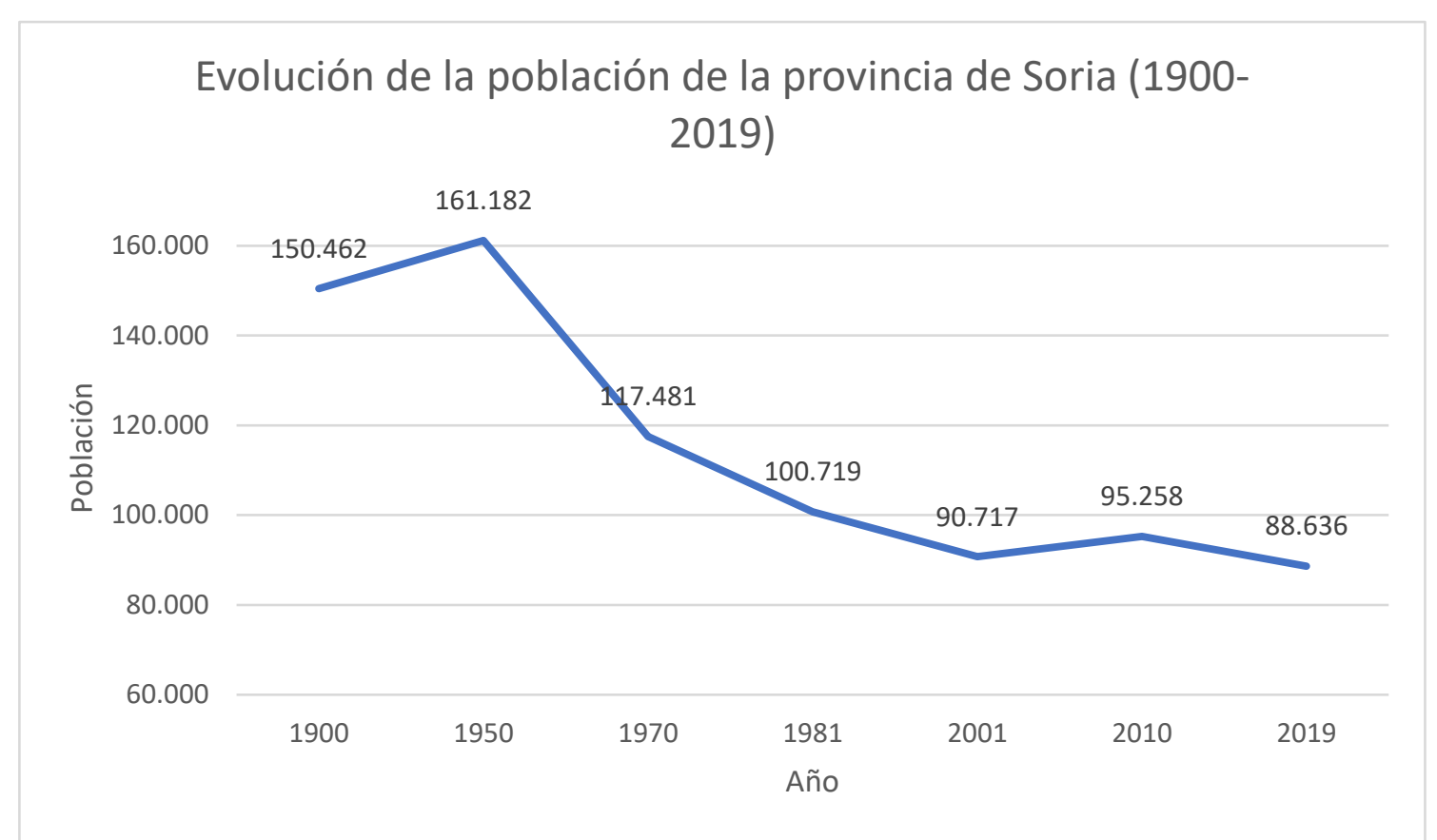

Fuente: elaboración propia a partir de datos del INE.

ANEXO 2: NÚMERO DE HABITANTES MAYORES DE 65 AÑOS EN LA PROVINCIA DE SORIA A 1 DE ENERO DE 2019 (POR FRANJAS DE EDAD).

\begin{tabular}{|c|c|c|c|}
\hline EDAD & HOMBRES & MUJERES & \\
\hline 65 a 69 & 2.555 & 2.400 & \\
\hline 70 a 74 & 2.274 & 2.110 & \\
\hline 75 a 79 & 1.818 & 2.031 & \\
\hline 80 a 84 & 1.596 & 2.192 & \\
\hline 85 a 89 & 1.379 & 1.997 & \\
\hline 90 a 94 & 614 & 1.127 & \\
\hline 95 a 99 & 155 & 394 & \\
\hline $\begin{array}{l}>100 \\
\text { años }\end{array}$ & 26 & 79 & \\
\hline TOTAL & 10417 & 12330 & $\begin{array}{r}22747 \\
(25,6 \%> \\
65 \text { años) }\end{array}$ \\
\hline
\end{tabular}

Fuente: elaboración propia a partir de datos del INE. 
ANEXO 3: LOCALIZACIÓN DE LOS CENTROS DE LOGOPEDIA INDICADOS EN EL REGISTRO GENERAL DE CENTROS, SERVICIOS Y ESTABLECIMIENTOS SANITARIOS DE LAS PROVINCIAS DE SORIA, SEGOVIA Y VALLADOLID.

Provincia de Soria

\begin{tabular}{lc}
\hline MUNICIPIO & NÚMERO DE CENTROS \\
\hline Soria & 3
\end{tabular}

Fuente: elaboración propia a partir de datos del Registro General de Centros, Servicios y Establecimientos sanitarios.

Provincia de Segovia

\begin{tabular}{lc}
\hline MUNICIPIO & NÚMERO DE CENTROS \\
\hline Segovia & 1 \\
\hline Cuéllar & 1
\end{tabular}

Fuente: elaboración propia a partir de datos del Registro General de Centros, Servicios y Establecimientos sanitarios

Provincia de Valladolid

\begin{tabular}{lc}
\hline MUNICIPIO & NÚMERO DE CENTROS \\
\hline Valladolid & 15 \\
\hline Olmedo & 1 \\
\hline Medina del Campo & 2 \\
\hline Arroyo de la Encomienda & 2 \\
\hline Tordesillas & 1
\end{tabular}

Fuente: elaboración propia a partir de datos del Registro General de Centros, Servicios y Establecimientos sanitarios 
ANEXO 4: MODELOS DE ENTREVISTA PARA EL ESTUDIO SOCIAL

\section{ENTREVISTA A LOGOPEDAS}

\begin{tabular}{|l|l|l|l|}
\hline Nombre del centro & & $\begin{array}{l}\text { Número de } \\
\text { logopedas }\end{array}$ \\
\hline $\begin{array}{l}\text { ¿Cuánto tiempo lleva } \\
\text { trabajando en la } \\
\text { provincia de Soria? }\end{array}$ & & Privado & \\
\hline Carácter del centro & \multicolumn{1}{|c|}{ Público } & & \\
\hline $\begin{array}{l}\text { Cantidad media de pacientes que } \\
\text { acuden al servicio a lo largo del año }\end{array}$ & & \\
\hline
\end{tabular}

1. ¿Cómo es la regularización existente de los pacientes para que acudan a su centro? (convenios existentes con la Seguridad Social, si acuden los pacientes a través de mutuas, asociaciones, los propios pacientes solicitan acudir a rehabilitación...

2. ¿Cuáles son las patologías que más acuden al servicio de logopedia en su centro?

3. ¿Realiza su trabajo exclusivamente en el centro? Si no es así, ¿a qué otros ámbitos acude? (Asociaciones, domicilio...)

4. ¿Qué aspectos potenciaría en cuanto a la regulación y organización de los pacientes de logopedia por parte del SACYL en la provincia de Soria?

5. ¿Existen aspectos deficitarios en el servicio de logopedia en la provincia de Soria con respecto a otras provincias o comunidades? ¿Qué aspectos mejoraría?

6. ¿influyen estos aspectos en el tratamiento a los pacientes? ¿Cómo? ¿Cuáles son los que más repercusiones tienen?

7. ¿Cuáles son las dificultades que más se repiten a la hora de que los pacientes lleven a cabo la rehabilitación?

8. ¿Cree que la logopedia es un servicio precario en la provincia de Soria? ¿Por qué?

9. Otros aspectos a comentar. 


\section{ENTREVISTA A FAMILIARES}

\section{Tenga en cuenta que todas las preguntas se refieren al servicio en general, NO al}

\section{tratamiento impartido por los/las profesionales.}

1. ¿Conocía el servicio de logopedia antes de que su familiar tuviera que acudir? Si es así, ¿por qué lo conocía?

2. ¿Qué supone o ha supuesto para usted el hecho de que su familiar acuda al logopeda? ¿Ha repercutido en su vida diaria (tiempo, implicación como familiar, coste económico adicional...)?

3. Como familiar que ha vivido el proceso desde un punto de vista externo, ¿qué destacaría del servicio de logopedia y de su organización? ¿Qué mejoraría?

4. ¿Cree que en la provincia de Soria la logopedia es un servicio precario? ¿por qué?

5. Desde su punto de vista como familiar, ¿qué necesidades existen, dentro del servicio de logopedia en la provincia de Soria, que impiden que sea $100 \%$ eficiente?

6. Cuente cómo ha vivido o cómo está viviendo el paso de su familiar por todo el proceso de intervención, desde que se produce la derivación o se plantea el acudir a logopedia, hasta el momento actual o la finalización del tratamiento.

7. Otros aspectos que quiera destacar o comentar sobre el servicio de logopedia en la provincia de Soria. 


\section{ENTREVISTA A PACIENTES}

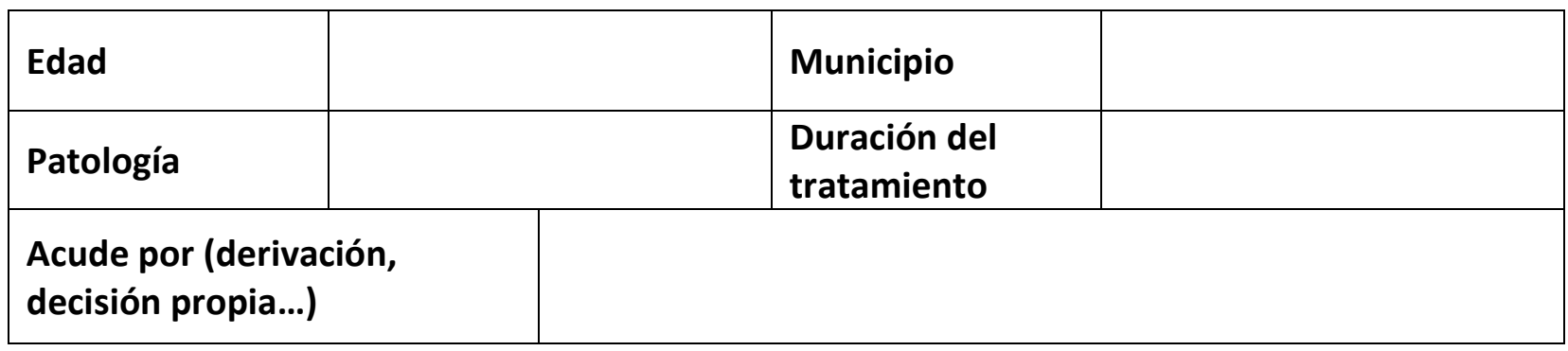

Tenga en cuenta que todas las preguntas se refieren al servicio en general, NO al tratamiento impartido por los/las profesionales.

1. ¿Conocía el servicio de logopedia antes de tener que acudir? Si es así, ¿por qué lo conocía?

2. ¿Qué supone o ha supuesto para usted ir al logopeda?

a) ¿Cuánto tiempo le dedicaba o le dedica a lo largo del día? (Incluyendo si tenía que realizar desplazamiento para acudir a las sesiones)

b) ¿Le supone o suponía un coste económico adicional tener que acudir a la sesiones?

c) ¿El tener que acudir/realizar el tratamiento suponía algún tipo de repercusión en su vida diaria? ¿Cuáles?

3. En general, ¿qué destacaría dentro del servicio y su organización? ¿qué mejoraría?

4. ¿Cree que esos puntos débiles han podido influir de alguna manera en el tratamiento

5. Si pudieras cambiar algo del servicio, ¿qué sería?

6. ¿Considera que existen diferencias con otros servicios de rehabilitación? (Fisioterapia, por ejemplo). ¿Podría indicar alguna?

7. Desde su punto de vista, ¿qué necesidades tiene el servicio de logopedia en la provincia de Soria para que sea $100 \%$ eficiente?

8. ¿Cree que la logopedia es un servicio precario en la provincia de Soria? ¿Por qué?

9. Cuente cómo ha vivido o como está viviendo su paso por todo el proceso de intervención, desde que se produjo la derivación o se planteó el acudir a logopedia, hasta el momento actual o la finalización del tratamiento.

10. Otros aspectos a destacar o comentar sobre el servicio de logopedia en la provincia de Soria. 